\title{
Antibiotic use in patients admitted with acute exacerbations of chronic obstructive pulmonary disease
}

\author{
J.A. Smith, P. Redman, M.A. Woodhead
}

\begin{abstract}
Antibiotic use in patients admitted with acute exacerbations of chronic obstructive pulmonary disease. J.A. Smith, P. Redman, M.A. Woodhead. (C)ERS Journals Ltd 1999. ABSTRACT: The objective of this report was to document the pattern of initial antibiotic prescribing in acute exacerbations of chronic obstructive pulmonary disease (COPD) in a hospital setting.

All episodes of acute exacerbation of COPD, as diagnosed by the admitting doctor, in one hospital in the period January to May 1996, were identified. Case notes were reviewed retrospectively. Cases of radiographic pneumonia, bronchiectasis and incorrectly coded admissions were excluded. Symptoms, microbial cultures and initial antibiotic therapies were recorded.

One hundred and fifty-nine patient episodes were identified; 40 were excluded yielding a sample of 119 . Nineteen case notes were unavailable leaving a sample of 100 (84\%) episodes. Eighty were treated with antibiotics on admission; amoxycillin was the most frequently prescribed, in $46(58 \%)$ episodes. Of the antibiotic treated group, $42(53 \%)$ patients were given dual therapy, most commonly a macrolide antibiotic with either amoxycillin or a cephalosporin. Intravenous treatment was used in 22 $(28 \%)$ cases. The duration of intravenous treatment was $>48 \mathrm{~h}$ in $12(15 \%)$ cases. A total of 76 sputum samples were analysed from 55 patient episodes: $34(45 \%)$ were culture positive. In $15(27 \%)$ patient episodes, antibiotic therapy was changed or instituted on the basis of culture results.

These data suggest that antibiotic treatment is not optimal, with overuse of antibiotics, especially intravenous and dual therapy.
\end{abstract}

Eur Respir J 1999; 13: 835-838.

Depts of Respiratory Medicine and Clinical Audit, Manchester Royal Infirmary, Manchester, UK.

Correspondence: M.A. Woodhead

Manchester Royal Infirmary

Oxford Road

Manchester M13 9WL

UK

Fax: 441612764989

Keywords: Antibiotic

chronic obstructive pulmonary disease guidelines

Received: June 251998

Accepted after revision December 81998
Bacteria are often cultured from the sputum of patients with acute exacerbations of chronic obstructive pulmonary disease (AECOPD), most commonly Haemophilus influenzae, Streptococcus pneumoniae and Moraxella catarrhalis. Despite this, the role of bacterial infections remains unclear. There are two main reasons to doubt their importance. Firstly, bronchoscopic studies show the presence of bacteria in similar numbers both between and during exacerbations, and the absence of bacteria in up to $50 \%$ during exacerbations [1-4]. Secondly, placebo controlled studies evaluating the effectiveness of antibiotic treatment in AECOPD show only a small benefit $[5,6]$. The study performed by ANTHONISEN et al. [5] examined the relationship between symptoms and response to antibiotic treatment. It showed that episodes of AECOPD which met three criteria (increased breathlessness, increased sputum volume and increased sputum purulence) were the most likely to benefit from antibiotics. They termed this a type 1 exacerbation. Type 2 exacerbations had any two of the three criteria and had less benefit from treatment. Type 3 exacerbations, with only one of the criteria, had no demonstrable benefit. There is a widespread perception that antibiotics are overused in AECOPD, but there is little detailed objective evidence to support this view.

The aim of this study was to document the initial antibiotic prescribing patterns for patients admitted to hospital with a diagnosis of AECOPD. The use of more than one antibiotic and the intravenous route of administration were of particular interest. The antibiotic policy of the authors' hospital at the time of the audit was to use oral amoxycillin (500 $\mathrm{mg}$ t.d.s.) in uncomplicated cases, tetracycline $(500 \mathrm{mg}$ q.d.s.) in the penicillin allergic group and co-amoxiclav if an antibiotic had been given recently.

\section{Subjects and methods}

Potential cases were identified retrospectively from the hospital's coding of acute admissions. The case notes of all episodes coded as having a primary diagnosis of AECOPD in the period January 1, 1996 to May 31, 1996 were examined. An episode was only included if the admitting doctor had also made a diagnosis of AECOPD. Cases found to have radiographic evidence of pneumonia or bronchiectasis were excluded. Patients admitted with AECOPD are not specifically admitted under the care of a respiratory physician, but rather by the general physician of the day who may or may not have a respiratory interest.

For each episode, respiratory symptoms (sputum colour, sputum volume and breathlessness) and antibiotic treatments were recorded and Anthonisen scores were calculated. For each antibiotic used, the route and duration of administration were documented. It was usual practice for antibiotic treatment decisions to be made following 
examination of the chest radiograph as this was almost immediately available. A patient was considered severely ill on admission if they were recorded as having an impaired conscious level, respiratory rate $>30$ breaths $\cdot \mathrm{min}^{-1}$ or if they required assisted ventilation. The results of microbiological examination of sputum, and their influence on management was also recorded.

Management was compared to the following standards. Patients with an Anthonisen type 1 exacerbation or who were severely ill should receive antibiotic treatment. It was an option for those with type 2 exacerbations, but type 3 exacerbations should not routinely receive antibiotics. The oral route should be used unless the patient is severely ill or vomiting. The maximum duration of antibiotic treatment should be no longer than seven days.

Sputum samples were cultured on blood and chocolate agar, both aerobically and anaerobically according to the normal laboratory protocol. Antibiotic resistance was measured using Stokes' method.

The antibiotic treated and nontreated groups were compared in terms of mean age using the Student's t-test and median duration of stay using the Mann-Whitney U-test. Previous antibiotic treatment, increased breathlessness, increased sputum purulence, increased sputum volume and management by a respiratory physician in each group was analysed using the two-tail Fisher's exact test.

\section{Results}

From the clinical coding 159 patient episodes were identified. Forty were excluded, most commonly on the grounds of miscoding. Of the 119 episodes fitting the study definition, 19 sets of notes were either missing or unavailable for review. The remaining sample of 100 (84\%) patient episodes, admitted under the care of 14 different physicians, was studied.

There were slightly more males $(n=57)$ than females $(n=43)$ and age distribution was not statistically different from the normal distribution (Kolmogarov-Smirnov test, $\mathrm{p}=0.2$ ), mean age of 67 yrs (range 38-90). Duration of admission was positively skewed (Kolmogarov-Smirnov test, $\mathrm{p}=0.008$ ) and the median length of stay was eight days (range 1-40). None of the patients were admitted to intensive care or received assisted ventilation; six patients died as a result of the exacerbation. The small size of this group meant that the impact of antibiotic treatment or sputum examination on outcome could not be assessed.

The main finding was that antibiotic treatment was given in 80 of the 100 episodes of AECOPD. In 42 episodes, more than half of those treated, a combination of two antibiotics were used. Figure 1 shows the commonest combinations of antibiotics.

Amoxycillin was the most frequently prescribed drug, but used most commonly in combination with erythromycin (fig. 1). Intravenous treatment was used in 22 episodes altogether, more than a quarter of those treated. From the information recorded in the case notes, none of those treated by the intravenous route met the criteria set in the standards. The overall median duration of inpatient antibiotic treatment was 5 days (range 2-20). For intravenous treatment, the median duration was 3 days (range $1-7$ ) and for those treated with two antibiotics, the second agent was continued for a median of 5 days (range 1-10). Previous

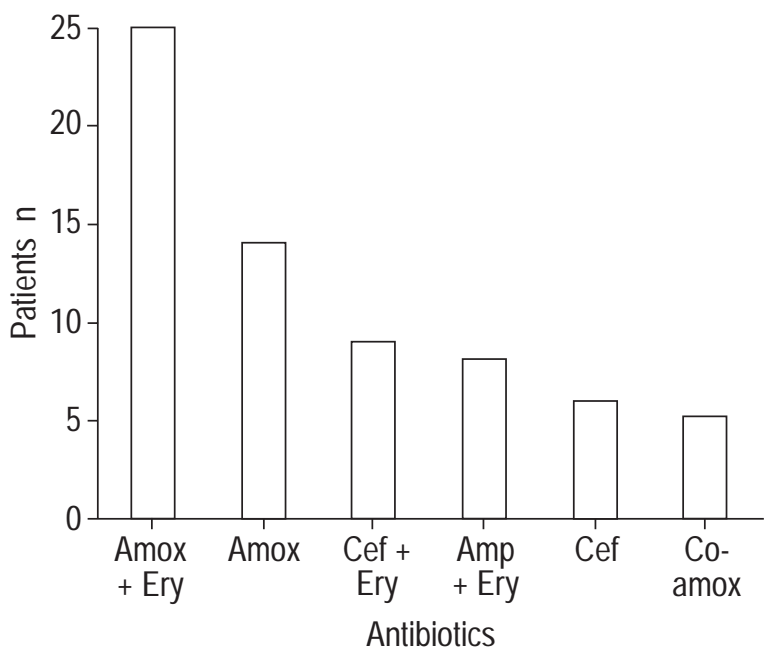

Fig. 1. - The most frequent initial antibiotic prescriptions used. Amox: amoxycillin; Ery: erythromycin; Cef: cefuroxime; Amp: ampicillin; Coamox: co-amoxiclav.

antibiotic treatment was known to have been given by the general practitioner in 36 episodes. In seven, it was recorded that no prior treatment had been given, but in 57 episodes this information was unrecorded.

Comparing the antibiotic treated group to the nontreatment group, the mean age was not significantly different (Student's t-test, $\mathrm{p}=0.89$ ). Although the median duration of admission was also not significantly different, there was a trend towards a shorter stay in the nontreatment group (Mann-Whitney U-test, $\mathrm{p}=0.057$ ). Table 1 shows previous antibiotic treatment and symptoms for both groups. Increased sputum volume and increased sputum purulence were significantly related to antibiotic use, although missing data makes interpretation difficult. Previous antibiotic treatment did not significantly influence whether or

Table 1. - Comparison of the number of patients in antibiotic treatment and nontreatment groups in relation to five features

\begin{tabular}{|c|c|c|c|}
\hline & Treatment & Nontreatment & p-value ${ }^{+}$ \\
\hline \multicolumn{4}{|c|}{ Previous antibiotic } \\
\hline Yes & 31 & 5 & 0.318 \\
\hline No & 5 & 2 & \\
\hline \multicolumn{4}{|c|}{$\begin{array}{l}\text { Increased breathless- } \\
\text { ness }\end{array}$} \\
\hline Yes & 79 & 20 & 1.000 \\
\hline No & 1 & 0 & \\
\hline \multicolumn{4}{|c|}{$\begin{array}{l}\text { Increased sputum } \\
\text { purulence }\end{array}$} \\
\hline Yes & 43 & 5 & 0.012 \\
\hline No & 32 & 15 & \\
\hline \multicolumn{4}{|c|}{$\begin{array}{l}\text { Increased sputum } \\
\text { volume }\end{array}$} \\
\hline Yes & 16 & 1 & 0.022 \\
\hline No & 24 & 14 & \\
\hline \multicolumn{4}{|c|}{$\begin{array}{l}\text { Managed by respira- } \\
\text { tory physician }\end{array}$} \\
\hline Yes & 18 & 16 & $<0.001$ \\
\hline No & 62 & 4 & \\
\hline
\end{tabular}

Sum of data for each item is $<100$ in some cases due to lack of documentation in case notes. ${ }^{+}$: Fisher's exact test. 
Table 2. - Number of patients in which symptoms were recorded

\begin{tabular}{lccc}
\hline & \multicolumn{3}{c}{ Symptoms } \\
\cline { 2 - 4 } & $\begin{array}{c}\text { Increased } \\
\text { breathlessness }\end{array}$ & $\begin{array}{c}\text { Increased } \\
\text { sputum } \\
\text { purulence }\end{array}$ & $\begin{array}{c}\text { Increased } \\
\text { sputum } \\
\text { volume }\end{array}$ \\
\hline Yes & 99 & 47 & 17 \\
No & 1 & 48 & 38 \\
Unknown & 0 & 5 & 45 \\
\hline
\end{tabular}

not an antibiotic was prescribed (Fisher's exact test, $\mathrm{p}=$ 0.318 ), nor was the pattern of prescribing (type of drug, drug combination or route) affected. Dual therapy was not significantly related to previous antibiotic treatment $(\mathrm{p}=$ 0.685 ). There was a significantly lower rate of antibiotic use by physicians with an interest in respiratory medicine as compared to those with interests in other medical specialities.

Calculation of Anthonisen scores for each episode was hampered by lack of recorded information. Changes in sputum volume were the most common omission (table 2). Scores could only therefore be calculated for 54 of the 100 patient episodes (fig. 2). It is suspected that negative findings are less often recorded and so this group is probably not representative of the whole. It does show however that the patients with the bulk of type 1 and 2 exacerbations were receiving antibiotics, whereas the majority who were not treated were type 3 . Of the episodes which could be scored, only $17(32 \%)$ met with the hospital antibiotic guidelines.

In 55 of the 100 patient episodes, sputum samples were sent for examination. A total of 76 samples were collected, from which a predominant bacterium was isolated in 34 $(45 \%)$. More than one organism was isolated from some samples giving 37 organisms cultured (fig. 3).

As expected, the commonest organisms were $H$. influenzae, S. pneumoniae and M. catarrhalis. Amoxycillin resistance was found in five organisms (two M. catarrhalis and three $H$. influenzae), Pseudomonas species were cultured in five samples, the significance of which is un-

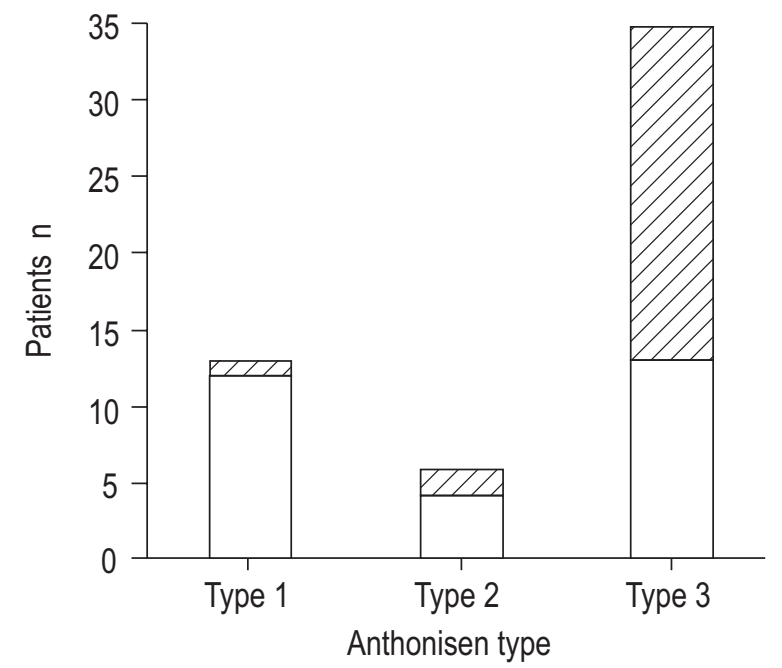

Fig. 2. - Antibiotic prescribing in relation to Anthonisen scores. $\square$ : antibiotics; $\mathbb{Z}$ : no antibiotics.

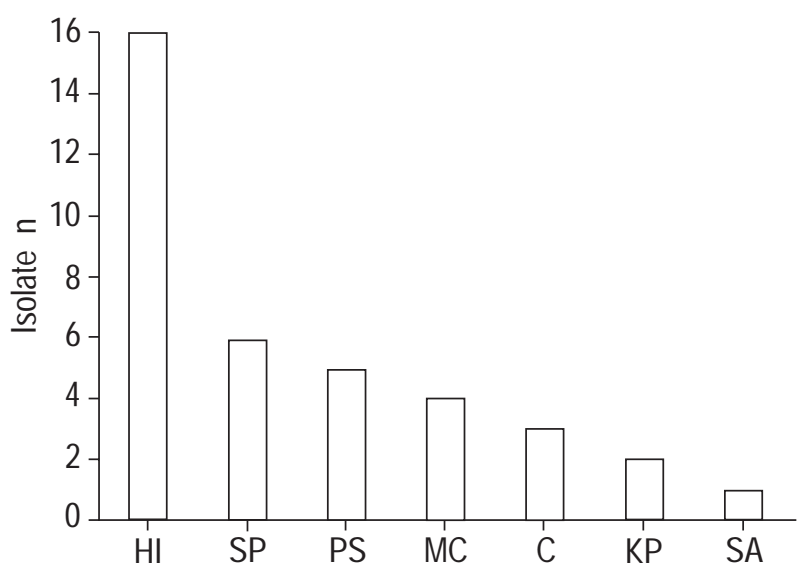

Fig. 3. - Bacteria cultured from sputum samples. HI: Haemophilus influenzae; SP: Streptococcus pneumoniae; PS: Pseudomonas spp., MC: Moraxella catarrhalis; C: coliforms; KP: Klebsiella pneumoniae; SA: Staphylococcus aureus.

certain. In $15(15 \%)$ of the episodes, antibiotic treatment was changed as a result of cultures. The appropriateness of these changes is difficult to judge retrospectively owing to the limited information in the case notes.

\section{Discussion}

This study suggests that at least in this hospital, antibiotics are being overused in the treatment of AECOPD. The precise place of antibiotics in the management of AECOPD is controversial and largely undefined. Objective evidence for their role is largely confined to the studies cited in the introduction and their findings are broadly reflected in the European Respiratory Society (ERS) guidelines for the treatment of lower respiratory tract infections [7] and British Thoracic Society (BTS) guidelines for the treatment of COPD [8]. Dual antibiotic treatment has no place in either document. It is believed that dual treatment was given as a result of the admitting doctor failing to distinguish between the treatment of simple AECOPD and the treatment of severe community-acquired pneumonia. The use of a $\beta$-lactam together with a macrolide, as recommended for severe community-acquired pneumonia $[7,9]$, occurred in all instances of dual therapy, supporting this view. It could be argued that treatment was started based on a presumptive diagnosis of severe pneumonia prior to viewing of the chest radiograph, however, chest radiographs are almost immediately available on admission. If antibiotics were prescribed without the benefit of the radiograph, it would be expected that dual therapy would be discontinued once this was obtained. This is not corroborated by a mean duration of dual therapy of five days; only two patients had the second antibiotic discontinued after only $24 \mathrm{~h}$.

The role of intravenous therapy is again unclear, but would be appropriate for severely ill patients. None of these patients met the given criteria for severe illness, none were admitted to the intensive care unit and there were just six deaths, suggesting that this group represents mainly nonsevere exacerbations. The use of intravenous treatment in $22 \%$ would therefore seem excessive. 
The main weaknesses of this study are its retrospective approach and its localization in just one hospital. Furthermore, a proportion of the notes were missing and there was a dependence on a correct coding to ensure that no episodes had been missed. It is unlikely that season affects the pattern of prescribing even though it affects the number of admissions. The overall sample size was large, but the size of the group not given antibiotics was relatively small, which affects the validity of comparisons with the group on antibiotics. The sample did not include tertiary referrals and appeared to be broadly typical of AECOPD patients across the country. It is not certain how well a sample limited to one group of junior doctors in one large metropolitan teaching hospital correlates to other settings. It is suspected that prescribing behaviour is similar to that in many other units and it is believed that the results confirm and quantify a major problem. This level of antibiotic use may not only be leading to increased antibiotic side-effects (although these were not assessed in this study), but also has significant financial implications.

The hospital standards used compared well with the recently published ERS guidelines for the treatment of lower respiratory tract infections [7] and the BTS guidelines for the treatment of COPD [8]. Although a hospital antibiotic policy was in place at the time of the audit it was not well publicised. In the light of these results, a new policy has been introduced, including indications for antibiotic treatment as well specifying drugs. This audit was presented at the hospital audit meeting and the guidelines have been made available on wards and the admission unit in an attempt to improve practices. It is intended to repeat the audit following these measures.

Further research is clearly needed to identify markers which will provide clearer indications for use of antibiotics in acute exacerbations of chronic obstructive pulmonary disease. In the authors' opinion the results of this study suggest overprescribing. If these results can be generalized, they may have important implications for the increasing frequency of antibiotic resistant organisms.

\section{References}

1. Cabello H, Torres A, Cells R, et al. Bacterial colonisation of distal airways in healthy subjects and chronic lung disease: a bronchoscopic study. Eur Respir J 1997; 10: 1137-1144.

2. Monso E, Ruiz J, Rosell A, et al. Bacterial infection in chronic obstructive pulmonary disease. Am J Respir Crit Care Med 1995; 152: 1316-1320.

3. Fagon J, Chastre J, Trouilet J, Domart Y, et al. Characterisation of distal bronchial microflora during acute exacerbation of chronic bronchitis. Am Rev Respir Dis 1990; 42: 1004-1008.

4. Soler N, Torres A, Ewig S, et al. Bronchial microbial patterns in severe exacerbations of chronic obstructive pulmonary disease (COPD) requiring mechanical ventilation. Am J Respir Crit Care Med 1998; 157: 1498-1505.

5. Anthonisen NR, Manfreda J, Warren CP, Hershfield ES, Harding GK, Nelson NA. Antibiotic therapy in exacerbations of chronic obstructive pulmonary disease. Ann Intern Med 1987; 106: 196-204.

6. Saint S, Bent S, Vittinghoff E, Grady D. Antibiotics in chronic obstructive pulmonary disease exacerbations. A meta-analysis. JAMA 1995; 273: 957-960.

7. European Study on community-acquired pneumonia Committee. Guidelines for management of adult community-acquired lower respiratory tract infections. Eur Respir J 1998; 11: 986-991.

8. The COPD Guidelines group of the Standards of care committee of the BTS. BTS guidelines for the management of chronic obstructive pulmonary disease. Thorax 1997; 52: Suppl. 5, S1-S28.

9. British Thoracic Society. Guidelines for the management of community acquired pneumonia in adults admitted to hospital. Br J Hospital Med 1987; 49: 346-350. 